\title{
EVALUACIÓN DE UN SISTEMA INSTRUCCIONAL AUTORREGULATORIO PARA UN AMBIENTE EN LÍNEA: EL CASO DE PSICOLOGÍA EN MÉXICO
}

\author{
(EVALUATION OF AN INSTRUCTIONAL SYSTEM FOR A SELF-REGULATORY ONLINE \\ ENVIRONMENT: THE CASE OF PSYCHOLOGY IN MEXICO)
}

\author{
Omar Moreno Almazán \\ Universidad Nacional Autónoma de México (México)
}

\section{RESUMEN}

El presente trabajo expone una experiencia que surgió en el Sistema de Universidad Abierta y Educación a Distancia (SUAED) en la Universidad Nacional Autónoma de México (UNAM) en el hecho de que en nuestro país surgió la iniciativa de ofrecer algunas licenciaturas en la modalidad de la educación a distancia, con el concepto del e-learning; entre estas licenciaturas está la educación superior en psicología. El surgimiento de la carrera fue en el año 2006 y para entonces comenzó un crecimiento que, entre otros problemas, desembocó en un desorden en la dosificación de contenidos temáticos, ausencia de orden vertical entre las asignaturas, así como el reflejo de la inexistencia de habilidades de aprendizaje autorregulado en la variada población estudiantil. Uno de los esfuerzos se encaminó a diseñar nuevas formas de dosificación y habilitación de estrategias de aprendizaje a partir de modelos cognoscitivos de autorregulación; los resultados se exponen en este documento.

Palabras clave: diseño instruccional, aprendizaje autorregulado, educación a distancia, psicología.

\begin{abstract}
This paper presents an experience that emerged in the System Open University and Distance Education at the National Autonomous University of Mexico (UNAM) which was a program initiated by Ecuador to provide some degrees through distance education, thus developing the concept of e-learning among universities in Mexico which offer higher education degrees in psychology. The beginning of the process was in 2006. At that time there began a growth which, among other problems, led to a disorder in the quantity of thematic content and an absence of vertical order between subjects which reflected the lack of skill of self-regulated learning in diverse student population. One of the efforts was aimed at improving the organization of material as well as promoting learning strategies based on cognitive models of self-regulation. The results are presented in this document.
\end{abstract}

Keywords: instructional design, self-regulated learning, distance education, psychology. 
Desde que Bandura introduce el concepto de autorregulación en su Teoría de Aprendizaje Social, en 1971, considerando además que en principio el individuo aprende a ejecutar las conductas mediante el aprendizaje que hace al observar de otros a través de un modelamiento basado en condicionamiento operante y que posteriormente el individuo ya no requiere más de reforzadores externos ni de modelos; es decir, el individuo es regulado externamente en una primera fase y poco a poco internaliza esta regulación para finalmente ser capaz de regularse por sí mismo. Entonces los teóricos en el estudio del aprendizaje autorregulado han generado modelos que nos permiten determinar el proceso, los rasgos, sustratos y elementos específicos que se deben considerar para su pleno entendimiento.

Entre lo más destacado se encuentra la idea de que los estudiantes pueden ser los principales promotores de sus procesos de aprendizaje y rendimiento académico; para ello y de acuerdo con Zimmerman (1986), los alumnos deben poner en práctica una serie de estrategias cognitivas, metacognitivas, motivacionales y conductuales. Así con el uso de estas estrategias el alumno puede construir un aprendizaje significativo, y además llevar un autoconocimiento acerca de las formas que cada uno tiene para utilizar y aplicar lo aprendido.

Otro de los puntos importantes es que los alumnos pueden regular su aprendizaje en tres dimensiones distintas: la cognición, la motivación y la conducta observable. El ámbito cognitivo implica el manejo de estrategias cognitivas y metacognitivas para realizar las tareas, además de que implica un conocimiento propio como procesadores de información y conocimiento de necesidades en términos de memoria, atención y conocimiento previo a fin de generar metas de aprendizaje más eficaces. En lo referente a la dimensión de motivación, es necesario que los alumnos logren controlar y hacer más realistas sus creencias personales sobre lo que son capaces de hacer en cada una de las tareas, identificando los distintos niveles de complejidad, así como las metas que pueden formularse ante cada situación de aprendizaje. Y a nivel conductual la autorregulación implica un cambio hacia una persona activa para crear ambientes que optimicen el propio aprendizaje, hallando modos y sitios adecuados de estudio y buscar ayuda de terceros (Pintrich, 1995).

A nivel de ambientes en línea, Azevedo, Winters y Moos (2004) señalan la importancia de que los alumnos utilicen habilidades autorregulatorias cuando están dentro de ambientes virtuales, en el hecho de que los hipertextos suelen estar en formatos no lineales y se ven obligados a regular su aprendizaje en lo motivacional, conductual y cognitivo para tomar decisiones respecto al establecimiento personal de metas, la información a acceder para lograr esas metas, la dosificación del tiempo 
para cada módulo o asignatura, la adecuación de estrategias de aprendizaje y su propio monitoreo de habilidades para la realización de tareas en distintos niveles de complejidad en trabajos individuales y colectivos entre otras. Sin embargo, son pocos los estudios que reportan los tipos de apoyo más eficaces para promover que los estudiantes que están en línea puedan regular sus procesos de aprendizaje en contextos virtuales; es por eso que uno de los elementos que puede apoyar al desarrollo de estas habilidades, es la implementación de programas de aprendizaje basados en los modelos de autorregulación.

Uno de los elementos que se han propuesto para el desarrollo de estas habilidades es la incorporación de escenarios de aprendizaje que sea regulado por un tutor o asesor que sea diseñador, orientador y dinamizador del proceso de aprendizaje; estudiantes que se sientan comprometidos y responsables de su propio proceso y un ambiente virtual que contenga elementos de apoyo para que el estudiante sea capaz de desarrollar su capacidad autorreguladora, utilizando sus propias metas. Un modelo de acción es el sugerido por López Vargas (2008) en donde propone un ambiente de aprendizaje computacional que se constituye principalmente de:

- Autoevaluación de los alumnos con respecto a sus tareas de aprendizaje.

- Establecimiento de metas.

- Estrategias de aprendizaje.

- Monitoreo de aprendizaje.

Esta propuesta es en sí la combinación de agentes artificiales con sistemas hipermedias, con el fin de generar el control en el ambiente respecto a la ejecución y toma de decisiones de los estudiantes. El desarrollo generó resultados en cuanto a la ejecución aunque plantea la investigación en términos de desarrollo de aspectos cognitivos y de análisis de las diferencias individuales para la adquisición de habilidades autorregulatorias.

La presente investigación tiene por objetivo el análisis de las habilidades autorregulatorias en un grupo de alumnos que fueron incluidos en un programa de enseñanza en línea de las neurociencias, de la licenciatura de Psicología a distancia del SUAED-UNAM. Se analizan las implicaciones individuales, su efecto en la adquisición de estas habilidades y las relaciones que se dan entre las distintas variables implicadas. 


\section{MÉTODO}

\section{Participantes}

El estudio se desarrolló con 66 estudiantes de la carrera de Psicología del Sistema de Universidad Abierta y Educación a Distancia (SUAED) de la FES Iztacala. Este grupo de alumnos se inscribieron en el módulo de Métodos de evaluación en las neurociencias de comportamiento de segundo semestre. Se tomaron en cuenta los datos de aquellos alumnos que pudieron terminar por completo su semestre.

\section{Materiales}

El módulo utilizó materiales diseñados exclusivamente para el módulo de Métodos de evaluación en las neurociencias del comportamiento del SUAED Psicología. El material que se empleó fue la antología del módulo con una guía de lectura y la integración digitalizada de cada artículo en formato de PDF Acrobat.

Se construyeron instrumentos de evaluación académica con la integración de ítems para las tres unidades: 1. Métodos de evaluación en las neurociencias del comportamiento, 2. Tacto y dolor y 3. Movimientos y acciones. Estos instrumentos fueron sometidos a Jueceo y cuentan con niveles de confiabilidad aceptable.

Se diseñó una programación de actividades basada en modelos instruccionales de Merrill (2002) con condiciones y actividades a desarrollar basadas en modelos psicopedagógicos y de evaluación basadas en Análisis Cognitivo de Tareas (ACT). El sustento psicológico de la programación tiene base en la psicología cognitiva, particularmente de los modelos de aprendizaje autorregulado de Boekaerts (2000) y de Pintrich (2000).

\section{CUVED}

El CUVED es un consorcio integrado por distintas entidades universitarias y diversas disciplinas, que tiene como fin, realizar servicios profesionales de evaluación, enseñanza y diagnóstico, entre otras. Se recurre al uso de plataformas virtuales donde es posible crear programas y modelos curriculares, que sirven para articular la educación tanto presencial como a distancia. Se tiene un ambiente virtual desarrollado por el CUVED, donde los estudiantes de esta investigación tuvieron su módulo al cual ingresaban mediante una clave de usuario y contraseña, e ingresaban al ambiente del módulo, donde encontraban la programación de actividades y los 
distintos recursos y tareas a realizar, así como el espacio para la interacción y envío de trabajos.

El CUVED mantenía, además, el registro de cada acción de los integrantes del grupo y permitía el ingreso a los elementos instruccionales:

- Libros electrónicos. Uno de los recursos que ofrecen las plataformas Moodle y que incorpora CUVED en ellos. Es posible generar materiales sencillos pero que contengan información básica sobre procesos o manuales. Incluían presentación, datos, procesos de evaluación, materiales multimedia y espacios de retroalimentación.

- Actividades colaborativas, en las cuales los alumnos eran integrados por equipos al azar para el desarrollo de ciertas tareas en donde se les exponían casos y problemas a resolver. Además del uso de foros, se emplearon recursos como chats y consultas con el fin de intercambio de información a nivel de discusión, organización y solución de problemas.

- Dinámica tutorial. La aplicación de distintos mecanismos de seguimiento y asesoría directa con los alumnos a nivel crónico y asincrónico. Se usaron desde espacios públicos y hasta la asesoría y asistencia personalizada mediante el uso de mensajería interna, y fue utilizado para la impartición de asesoría, supervisión y conducción. La comunicación con el alumno se realizaba en periodos de respuesta de entre 1 y hasta 5 días como máximo.

También se incluyeron en el CUVED espacios de intercambio de información entre los alumnos, mediante foros abiertos y mensajería intragrupos. Un espacio de integración de sus cronogramas de actividades basados en los objetivos del módulo y adecuados a las metas individuales con posibilidad de consulta constante, el envío de notas en actividades, y agenda y automonitoreo. 


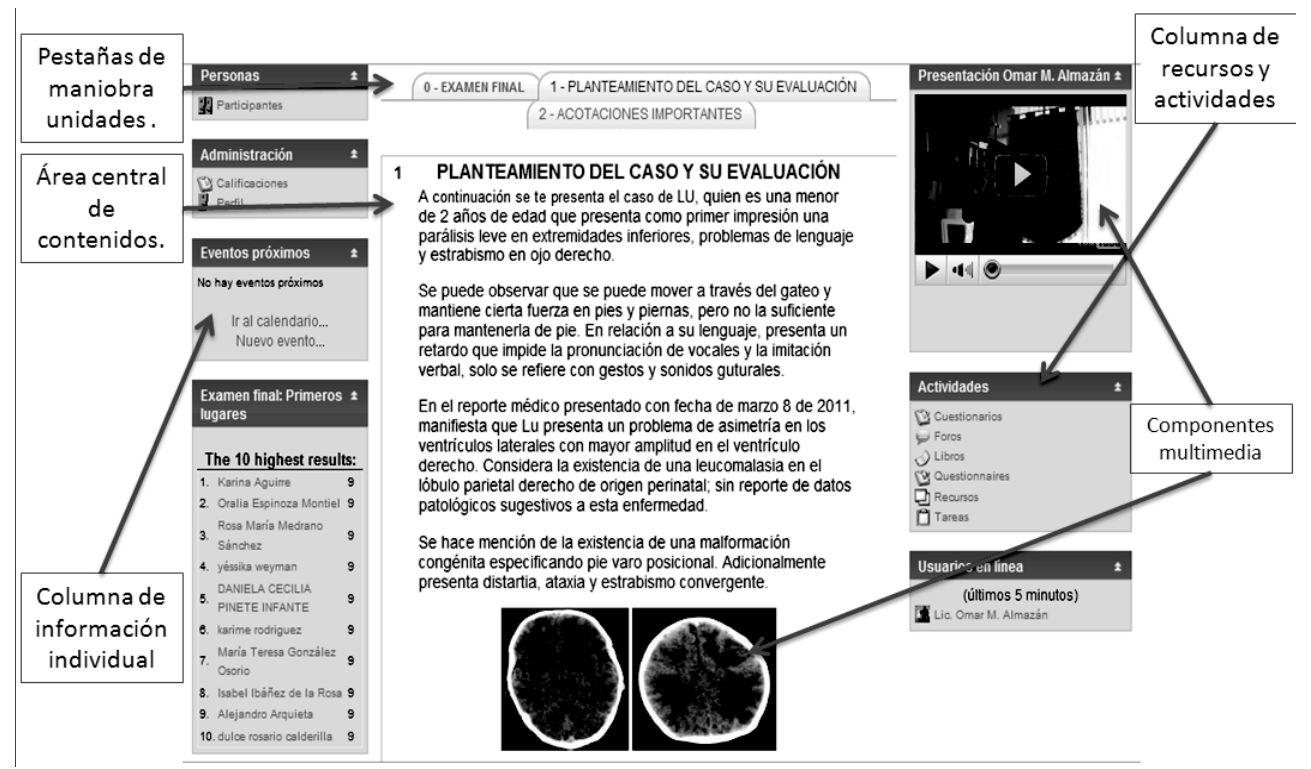

Figura 1. Vista del ambiente virtual del módulo en CUVED

El área de aprendizaje del CUVED se construye de un área central de contenidos en donde es posible visualizar las actividades centrales de trabajo en cada unidad, convirtiéndose en el espacio general de trabajo. Estas áreas se consultan y pueden ser cambiadas de acuerdo al interés del alumno, esto se hace mediante las pestañas de maniobra de las unidades. A los lados hay dos columnas, en la del lado derecho se encuentran los recursos y actividades que el alumno puede usar, y es el punto de referencia y apoyo del alumno. La columna del lado izquierdo, contempla espacios de consulta, administración e información para el alumno. Este ambiente virtual incluye, además, algunos recursos virtuales basados en la multimedia o en materiales de audio o visuales (Véase figura 1).

\section{Instrumento}

Se aplicó el Inventario de Estilos de Aprendizaje y Orientación Motivacional (EDAOM) en su versión en línea, validada y estandarizada para poblaciones mexicanas. Es un instrumento que evalúa principalmente las estrategias de aprendizaje y las orientaciones motivacionales del estudiante, fundamentado en las nociones centrales de la actividad cognitiva constructiva conocida como estudio (Castañeda y Martínez, 1999); está estructurado a partir de dos secciones, la de autorreporte y la de ejecución. Este instrumento identifica las autovaloraciones 
que los estudiantes realizan sobre sus estrategias de aprendizaje y orientaciones motivacionales al estudio. Constituido por 91 reactivos tipo Likert, organizados en 4 escalas que evalúan: 1) Estilos de adquisición de la información, con estrategias en dos niveles de procesamiento: a) selectivas y b) generativas; 2) estilos de recuperación de la información aprendida, en dos contextos: a) ante diferentes tareas académicas y b) durante los exámenes; 3) estilos de procesamiento, constituidos por: a) convergente y b) divergente; 4) estilos de autorregulación metacognitiva y metamotivacional, constituidos por dos componentes: a) los de la persona (eficacia percibida, contingencia interna, autonomía percibida, orientación a la aprobación externa), y b) los de los materiales de aprendizaje, en cuanto a su utilidad para propiciar el aprendizaje eficiente.

El EDAOM ha sido validado con aplicación a 2, 995 estudiantes de distintas instituciones educativas del país. Se determinó su validez concurrente en 0.67 promedio general de calificaciones, y 0.89 en tareas académicas diversas. Su consistencia interna es de 0.94 para todo el instrumento (alfa de Cronbach).

También se ha establecido la validez convergente y divergente de los constructos subyacentes mediante el análisis factorial confirmatorio (Castañeda y Ortega, 2004).

\section{Procedimiento}

Los alumnos fueron ingresados y registrados en la plataforma virtual del CUVED, y tuvieron una presentación en vídeo por parte del tutor. Para poder realizar las actividades del módulo debieron, primero, conocer la plataforma a fin de adaptarse a la misma y desarrollar un perfil de identidad personal y universitaria. Se les presentó el contenido temático en una programación que dosificó actividades de la manera en la que se presenta en la tabla 1.

\begin{tabular}{cc|} 
fases & Actividades \\
\hline $\mathbf{1}$ & Actividad inductiva \\
\hline $\mathbf{2}$ & Unidad 1 \\
\hline $\mathbf{3}$ & Actividad colaborativa unidad1 \\
\hline $\mathbf{4}$ & Tarea 1 \\
\hline 5 & Unidad 2 \\
\hline
\end{tabular}




\begin{tabular}{cc} 
fases & \multicolumn{1}{c}{ Actividades } \\
\hline 6 & Actividad colaborativa unidad 2 \\
7 & Tarea 2 \\
\hline $\mathbf{8}$ & Unidad 3 \\
$\mathbf{9}$ & Actividad colaborativa unidad 3 \\
\hline $\mathbf{1 0}$ & Tarea 3 \\
\hline $\mathbf{1 1}$ & Evaluación sumativa final \\
\hline $\mathbf{1 3}$ & Evaluación formativa \\
\end{tabular}

Tabla 1. Estructura de actividades modulares

Como se puede observar, la estructura general del módulo comienza a dosificar la presentación temática con actividades individuales y colectivas estructuradas con las bases que se señalaron. Cada participante tenía acceso a los contenidos temáticos, recursos y medios de evaluación y comunicación en el momento que dispusieran. Aunque las fechas de realización y avance en cada área estaba determinado por cada alumno, el diseño instruccional se mantuvo constante durante todo el semestre.

\section{Actividades de aprendizaje autorregulado}

La programación de actividades contó con estrategias cognitivas y pedagógicas que permitía una serie de opciones de trabajo basado en los modelos de autorregulación, a saber:

- Perfil personal: donde el alumno debía brindar cierta información sobre su persona en dos niveles. Como individuo en cuanto a descripciones generales y principales características, y, como miembro universitario, brindando información en cuanto a su percepción y objetivos como miembro de la UNAM. Debía incluir una foto reciente. 
- Cronograma de actividades: en el cual el alumno establecía, con base a la estructura del módulo, los tiempos y niveles de avance que él desarrollaría para cada actividad. En esta estructura se incluyó un calendario de avance y el establecimiento de metas curriculares. Este cronograma podía ser revisado por el tutor y por el alumno de manera permanente.

- Recursos virtuales específicos: estos recursos se expresaron en términos de resultados esperados y obtenidos, además de que contaron con una valoración numérica.

- Actividades inductivas: en las cuales los alumnos hacían gala de su conocimiento previo y podían utilizarlo para estimar su nivel de avance y sus necesidades de conocimiento.

- Biblioteca digital: un sitio universitario permanente en el cual el alumno podía acceder a bases de datos y documentos electrónicos selectos para su consulta y apoyo formativo.

- Autoevaluación: documento que permitió a los alumnos evaluar su propio rendimiento y avance a partir de sus calificaciones y del cumplimiento de metas al final del módulo.

El desarrollo del semestre se efectuó en un sistema completamente basado en el e-learning en donde el tutor se localizó en un espacio geográfico y los alumnos estaban también en sus respectivas entidades, no habiendo en ningún momento presencialidad o sincronía temporal con el grupo.

Al término de las actividades sumativas y formativas, los alumnos respondieron el cuestionario EDAOM con el fin de obtener los niveles de autorregulación en el grupo mediante una muestra representativa. Este cuestionario también se incluyó en línea y fue dispuesto a los alumnos para que lo respondieran después de haber sido completamente evaluados.

\section{RESULTADOS}

Uno de los primeros indicadores que se tienen es averiguar respecto a la incidencia que los alumnos tienen hacia cada una de estas actividades autorregulatorias, con el fin de ver el apego y adaptación de los alumnos en un sistema de enseñanza en línea con actividades autorregulatorias. En la figura 1 es posible ver la proporción en 
cuanto al uso que los alumnos le dieron a cada una de las actividades de aprendizaje autorregulado.

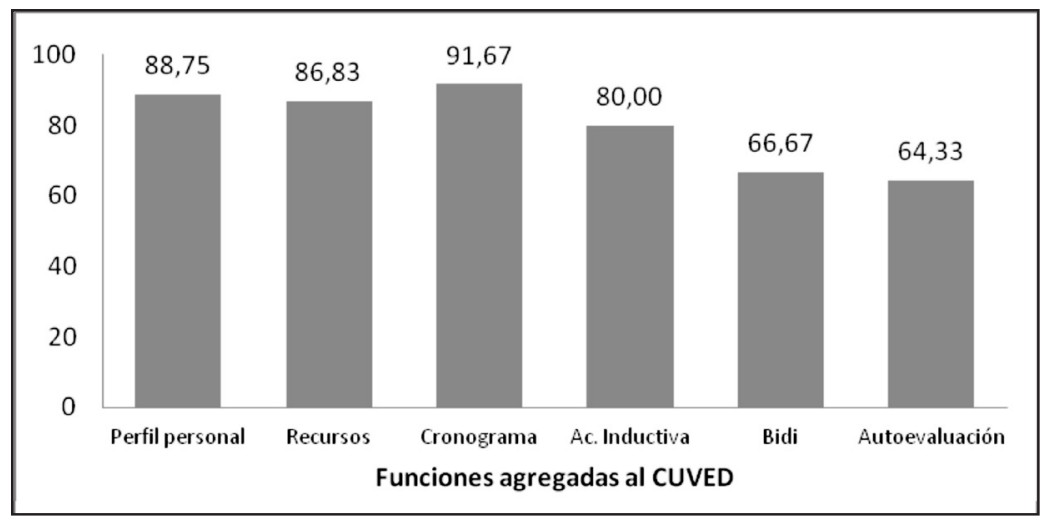

Gráfico 1. Porcentaje de usuarios en actividades de regulación

En el gráfico se expone la proporción de alumnos que hicieron uso de estos recursos y que además lo reflejaron en el desarrollo de sus actividades. Es posible observar que el $86.83 \%$ de los alumnos hicieron uso de los recursos que se ofrecieron en la plataforma para un mejor control y avance de su aprendizaje, pero además que el 88.75\% de ellos trabajaron tras la realización de un perfil personal que les daba individualidad ante su grupo y una imagen como miembro universitario. Lo más sobresaliente es que el $91.67 \%$ de ellos hizo uso del cronograma de actividades.

Los recursos que tuvieron menor uso fueron la biblioteca digital de la UNAM, que la usaron el $66.67 \%$ de los alumnos, y la autoevaluación que hicieron al final, donde el 64.33\% de los casos la utilizaron. Es importante señalar que ningún recurso tuvo un nivel de visitas o uso menor al 50\%, lo cual nos da un parámetro razonable en cuanto a la pertinencia de la inclusión de este tipo de actividades.

En cuanto al nivel de trabajo para las actividades tenemos los datos de la tabla 2.

\begin{tabular}{|c|c|c|c|}
\hline & $\begin{array}{l}\text { Frecuencia en el } \\
\text { uso de recursos }\end{array}$ & $\begin{array}{l}\text { Porcentaje de cumplimiento } \\
\text { de tiempos en cronograma }\end{array}$ & $\begin{array}{c}\text { Porcentaje de participación en } \\
\text { actividades formativas }\end{array}$ \\
\hline Media & 11.58 & 46.65 & 10.44 \\
\hline Mínimo & $\mathrm{O}$ & & o \\
\hline Máximo & 40 & & 32 \\
\hline
\end{tabular}

Tabla 2. Media, mínimo y máximo en cuanto al uso de los recursos autorregulatorios por semana 
Observamos en la tabla 2 algunos indicadores acerca de ciertos elementos de aprendizaje, entre lo que destaca que en cuanto a la frecuencia que los alumnos hacían uso de los recursos virtuales, en promedio, era de 11.58 visitas por semana, habiendo casos que no lo hacían, y otros tantos que llegaron a un punto máximo de 40 visitas a la semana de esos recursos. Para el caso de cumplimiento de los tiempos establecidos en sus propios cronogramas, tenemos que cerca del $46.65 \%$ de los alumnos que lo realizaron, cumplieron cabalmente con los tiempos. En cuanto a la proporción de visitas por semana de las actividades formativas, tenemos que estas eran de 10.44 visitas por semana, mientras que hubo personas que no llegaron a visitar dichas actividades, y otras que recurrían hasta 32 veces por semana a dichas actividades.

El uso de perfil es un elemento de identidad y da individualidad ante el grupo, además de fomentar su integración y motivación personal. Para este caso, tenemos los datos de la tabla 3 .

\begin{tabular}{|l|c|c|}
\hline & Frecuencia & Porcentaje \\
\hline No & 2 & 3.0 \\
Realización mínima & 33 & 50.0 \\
Realización aceptable & 15 & 22.7 \\
Si & 16 & 24.2 \\
Total & 66 & 100.0 \\
\hline
\end{tabular}

Tabla 3. Nivel de realización del perfil personal

Ya decíamos anteriormente que el uso del perfil había tenido impacto, sin embargo, de las personas que tuvieron actividad en el módulo tenemos que hubo dos quienes a pesar de haber trabajado, nunca realizaron un cambio en su perfil. Algunos de ellos (50\% de los casos) hicieron una realización mínima, lo cual indica que solo subieron una foto o dieron una descripción simple de su persona. El 22.7\% de ellos brindó información poco más abundante, y solo el 24.2\% de los casos se apegaron a los lineamientos que se solicitaron para la realización de este perfil.

En cuanto a los indicadores finales de autorregulación obtenidos en esta muestra tenemos algunos perfiles que nos brindan información respecto al desarrollo que los alumnos presentan cuando están dentro de un sistema con una programación diseñada con base a los modelos. Tras la aplicación del EDAOM se tienen indicadores que nos señalan el grado de adquisición de habilidades, mediante una estimación que va en los distintos niveles. 
En primer lugar tenemos los indicadores de los niveles de autorregulación en cuanto a frecuencia, vistos en la tabla 4.

\begin{tabular}{|l|c|c|}
\hline \multicolumn{1}{|c|}{ Subdimensión } & Media & Desv. típ. \\
\hline Eficacia Percibida Frecuencia & 67.24 & 20.005 \\
Contingencia Interna Frecuencia & 70.71 & 14.417 \\
Autonomía Percibida Frecuencia & 69.35 & 15.588 \\
Aprobación Externa Frecuencia & 17.94 & 10.286 \\
Logro de Metas Frecuencia & 72.24 & 11.670 \\
Tarea en sí Frecuencia & 55.29 & 14.687 \\
Materiales Frecuencia & 71.12 & 22.082 \\
\hline
\end{tabular}

Tabla 4. Indicadores terminales de habilidades de autorregulación en frecuencia

Se observa que los niveles de autorregulación en las subdimensiones señaladas siguen siendo considerados niveles bajos en términos de frecuencia, esto es, que la frecuencia con la que los alumnos usan estrategias para adquirir selectivamente la información a partir de un control generado por sí mismos es baja. Los niveles más altos corresponden a la frecuencia en el uso de materiales autorreguladores y en la frecuencia con la que perciben un logro de sus metas. Por su parte, entre los indicadores de menor medida se encuentra la frecuencia con la que los alumnos desarrollan tareas basadas en estos modelos (media de 55.29), y de manera aun más baja, la frecuencia con la que los alumnos se sujetan a la aprobación externa a fin de generar estrategias de autorregulación. Estas subdimensiones señalan la cantidad de veces a las que recurren los alumnos a las diversas formas de fomento de habilidades autorregulatorias.

Otro de los elementos corresponde a la facilidad con la que los alumnos son capaces de realizar con éxito las tareas y estrategias basadas en los modelos de autorregulación, teniendo los datos que aparecen en la tabla 5. 


\begin{tabular}{|l|c|c|}
\hline \multicolumn{1}{|c|}{ Subdimensión } & Media & Desv. típ. \\
\hline Eficacia Percibida Facilidad & 62.12 & 29.916 \\
Contingencia Interna Facilidad & 59.71 & 25.335 \\
Autonomía Percibida Facilidad & 68.82 & 24.370 \\
Aprobación Externa Facilidad & 20.12 & 17.574 \\
Logro de Metas Facilidad & 61.63 & 26.422 \\
Tareas en sí Facilidad & 52.88 & 26.122 \\
Materiales Facilidad & 68.82 & 23.343 \\
\hline
\end{tabular}

Tabla 5. Indicadores terminales de habilidades de autorregulación en facilidad

Se observan medias similares que nos indican qué tan fácil representa a los alumnos el desarrollo y adquisición de estas habilidades, teniendo principalmente que es para ellos menos fácil generar cambios cognitivos y conductuales propicios para la adquisición de habilidades regulatorias a partir de la aprobación de los demás, lo cual pudiera representar un problema el hecho de que la percepción de los demás hacia ellos es poco determinante para un cambio. Entre los rasgos de mayor nivel se ubican tanto la autonomía que los alumnos perciben que están adquiriendo por sí mismos para realizar sus cambios a nivel conductual y cognitivo, así como la facilidad que representó para ellos el uso de los materiales que se les proporcionó para este fin, ambos con una media de 68.82, lo cual es aceptable.

Un elemento más a ser considerado fue el del resultado que los alumnos obtienen al final de su trabajo, el cual se explica en la tabla 6.

\begin{tabular}{|l|c|c|}
\hline \multicolumn{1}{|c|}{ Subdimensión } & Media & Desv. Típ. \\
\hline Eficacia Percibida Resultado & 84.06 & 20.873 \\
Contingencia Interna Resultado & 82.35 & 15.716 \\
Autonomía Percibida Resultado & 79.88 & 21.598 \\
Aprobación Externa Resultado & 31.94 & 26.128 \\
Logro de Metas Resultado & 76.47 & 21.544 \\
Tareas en sí Resultado & 71.35 & 23.746 \\
Materiales Resultado & 81.47 & 23.136 \\
\hline
\end{tabular}

Tabla 6. Indicadores terminales de habilidades de autorregulación en resultado

Es aquí en donde los alumnos obtuvieron mejores puntajes, lo cual es considerado favorable en el sentido de que representa el logro y cumplimiento, así 
como la satisfacción de ellos hacia lo que obtuvieron al final de su proceso formativo. Tenemos entre los puntajes bajos que los resultados obtenidos tras el hecho de sujetarse a la aprobación de los demás son pobres (Aprobación externa en un promedio de 31.94); mientras que los índices más altos se ubican en los resultados favorables que ellos perciben tras el uso de estos materiales (Materiales en 81.47), en el manejo que dieron a su propio ritmo de estudio (Contingencia interna en 82.35), y en la autovaloración que se hacen a sí mismos como aprendices tras la obtención de su formación específica (Eficacia percibida en 84.06). Con estos datos es posible afirmar que los niveles obtenidos son favorables si tomamos en cuenta que los indicadores establecidos por el EDAOM mantiene una forma de interpretación como la que se señala en la tabla 7:

\begin{tabular}{|c|l|}
\hline Porcentaje & \multicolumn{1}{c|}{ Interpretación } \\
\hline 100 a 76 & No existe riesgo, hay un buen desarrollo de estrategias \\
\hline 75 a 56 & Se sugiere reforzar las estrategias pero no representa una falla crítica \\
\hline 55 a o & Necesidad crítica de entrenamiento, se considera falla significativa \\
\hline
\end{tabular}

Tabla 7. Interpretación del perfil en el EDAOM (Castañeda, 2004)

Uno de los elementos que también se considera importante es el desarrollo de habilidades autorregulatorias en ambientes de estudio en línea, los cuales presentaron los siguientes datos vistos en la tabla 8:

\begin{tabular}{|l|c|c|}
\hline \multicolumn{1}{|c|}{ Dimensión } & Media & Desv. típ. \\
\hline Dimensión estudio en línea Frecuencia & 16.53 & 4.823 \\
Dimensión estudio en línea Facilidad & 19.00 & 2.574 \\
Dimensión estudio en línea Resultado & 20.18 & 2.506 \\
\hline
\end{tabular}

Tabla 8. Indicadores terminales de habilidades de autorregulación en estudio en línea

Tomando en cuenta que la escala con la que se valora esta dimensión de estudio en línea es una adaptación del EDAOM, realizada por Peñaloza (Op. Cit.), y en la cual se puede considerar que el punto más bajo se ubicaría entre o y hasta 11, el punto medio entre 12 y 18, y el nivel alto es entre 19 a 21. Tenemos que para los alumnos inmersos en un programa de autorregulación en educación a distancia, recurren en menor medida proporcional a este estudio, con una media de 16.53 lo cual pudiera ser considerado como una parte habitual que los alumnos hacen con relación a los demás módulos, en el hecho de que ellos no asisten a clases presenciales. Mientras 
tanto, para ellos les resultó fácil (19) y con buenos efectos (20.18) el uso del estudio en línea en este programa.

Así, de manera comparativa tenemos un aumento significativo con relación a la media general (Tabla 9):

\begin{tabular}{cccccccc}
\hline & & \multicolumn{2}{c}{ Frecuencia } & \multicolumn{2}{c}{ Facilidad } & \multicolumn{2}{c}{ Resultado } \\
\cline { 2 - 8 } & & $\mathrm{U}^{*}$ & $\mathrm{G}$ & $\mathrm{U}$ & $\mathrm{G}$ & $\mathrm{U}$ & $\mathrm{G}$ \\
\hline \multirow{2}{*}{$\begin{array}{c}\text { Dimensión } \\
\text { estudio en línea }\end{array}$} & $\begin{array}{c}\text { Dimensión estudio en } \\
\text { línea }\end{array}$ & 5.83 & 16.53 & 13.17 & 19 & 12.42 & 20.18 \\
\hline \multirow{3}{*}{$\begin{array}{c}\text { Dimensión } \\
\text { persona }\end{array}$} & Eficacia percibida & 26.17 & 67.24 & 17.85 & 62.12 & 18.46 & 84.06 \\
\cline { 2 - 8 } & Contingencia interna & 22.87 & 70.71 & 30.42 & 59.71 & 11.29 & 82.35 \\
\cline { 2 - 8 } & Autonomía percibida & 22.17 & 69.35 & 32.13 & 68.82 & 20.29 & 79.88 \\
\cline { 2 - 8 } & Aprobación externa & 84.92 & 17.94 & 73.29 & 20.12 & 57.12 & 31.94 \\
\hline \multirow{2}{*}{\begin{tabular}{c} 
Dimensión tarea \\
\cline { 2 - 8 }
\end{tabular}} & Logro de metas & 20.58 & 72.24 & 35.71 & 61.63 & 19.63 & 76.47 \\
\cline { 2 - 8 } & Tarea en sí & 32.75 & 55.29 & 41 & 52.88 & 23.83 & 71.35 \\
\hline Dimensión & Materiales & 24.71 & 71.12 & 25.62 & 68.82 & 17.83 & 81.47 \\
\hline
\end{tabular}

* $U=$ Universo muestra de todo el SUAED; $G=$ Grupo de intervención de $2{ }^{\circ}$ semestre

Tabla 9. Comparativo entre las medias en EDAOM del universo y las del grupo de intervención

En la tabla 9 tenemos los datos que se han obtenido en el EDAOM al aplicarlo a una muestra de toda la población del SUAED (U) comparados con los datos que se tienen en el grupo de intervención (G), lo que hace posible identificar diferencias importantes. Vale resaltar que en todas las dimensiones existe una tendencia al alza con la excepción del correspondiente a la "Aprobación externa", en donde resulta ser el mejor puntaje para la población en general, y es en donde existe una reducción importante en el grupo de intervención. La explicación se centra en el hecho de que para la población en general resulta importante la percepción que los demás tienen acerca de su proceso formativo y dependen mucho de esa percepción; mientras que el grupo de intervención tiende a proyectar su motivación hacia la percepción interna y sus propias conductas y motivaciones, reduciendo el impacto que les pudieran generar los demás acerca de su trabajo.

Uno de los elementos que se buscó probar fue la independencia entre variables, utilizando la $J i$ cuadrada $\left(\mathrm{X}^{2}\right)$ de Pearson, en donde no se encontraron relaciones significativas entre las mismas con excepción de algunas condiciones. Uno de los valores observados de $X^{2}{ }_{(119)}$ fue de 157.56 con $p<0.05$ en la evidencia que apoya 
la suposición de que el resultado obtenido en las tareas en sí no es independiente al cumplimiento de tareas que los alumnos mostraron, de tal forma que estos hallazgos empíricos indican que la percepción que los alumnos tienen acerca de sus resultados en las tareas se relaciona con el cumplimiento que realizaron en el curso del semestre.

En este sentido podemos asegurar cómo el establecimiento de metas y la propuesta de resultados determinados permite al alumno moldear su forma de acción en el proceso de aprendizaje, dirigiendo, además, cada actividad formativa con índices de calidad que cada participante espera. En este sentido, y como señalan Fernández, Carballos y Delavaut (2008), el trabajo y aplicación que efectúan los alumnos para generar un autoaprendizaje gradual implica, entre otros componentes, congruencia entre la labor intelectual y la información de calidad, habilidades de autogestión (propósitos y establecimiento de metas), y tener los resultados vistos con cierta calidad que, en muchos casos, se ve reflejada en las calificaciones obtenidas. Así es posible determinar que los alumnos en sistemas de educación a distancia generan una labor de autoaprendizaje a partir de las metas que cada uno establezca en términos de la calidad del resultado final que pueden obtener.

Otra de las relaciones encontradas fue un valor observado de $X^{2}{ }_{(21)} q u e$ fue de 36.93, con $p<0.05$, en cuanto a que se indica que el resultado obtenido en las tareas está relacionado con el cumplimiento de los tiempos establecidos por cada uno en sus propios cronogramas de avance. Según Sarramona (1999), el éxito en la iniciativa y procesos de gestión en el aprendizaje está en manos del propio sujeto que aprende, sin excluir el papel del docente en sus funciones tutoriales y en la forma que da uso a la planeación y a los materiales que brindará. Con la planificación de actividades dosificadas en metas a corto plazo y secuenciadas de manera gradual, se puede promover un ejercicio de control de actividad por parte del alumno, y su impacto sobre el aprendizaje tiende a ser más significativo.

A su vez, se observa otra relación de $X^{2}{ }_{(10)} q u e$ fue de 18.58, con $p<0.05$, para el caso del resultado en el logro de metas y la eficiencia terminal del módulo; de tal forma que se apoya la idea de que los alumnos perciben un cumplimiento de metas que favorecen sus procesos de autorregulación a partir de su calificación final y el cumplimiento de objetivos tanto curriculares como personales en torno al módulo.

Este último hallazgo remite a la importancia de la motivación dentro del proceso de aprendizaje en entornos virtuales. Desde enfoques relativos al estudio de estos procesos a nivel cognitivo y conductual, destaca la importancia que tiene establecer metas que favorezcan el proceso, en donde los factores clave son el papel que 
desempeña el profesor desde el establecimiento de programación de contenidos y establecimiento de objetivos, y el papel del alumno en cuanto a tener los elementos que dirijan su trabajo en el logro (Amador, 1998). De esta forma es posible reiterar que el éxito en la formación de un alumno estará relacionado directamente con el hecho de brindarle los recursos para que él genere expectativas suficientes para tener resultados que tengan impacto a nivel personal y profesional; todo ello considerando que no existe en ningún momento una interacción personal y directa que favorezca la aparición de estos logros.

\section{DISCUSIÓN}

Si algo resulta importante señalar es que se puede evidenciar empíricamente que el desarrollo de programas de estudio en línea, basados en un modelo instruccional y de estilos de autorregulación, adecuados en un ambiente virtual de aprendizaje, propicia desempeños significativamente altos en las evaluaciones y en la permanencia de alumnos del sistema de educación a distancia. Los efectos de estos programas pueden tener explicaciones diversas que impactan en distintos niveles que van en planos motivacionales, conductuales, cognitivos y contextuales.

Generalmente existe la idea de que la inclusión de novedades tecnológicas en sí mismas pueden propiciar un mejor desempeño, sin embargo, tales argumentos pudieran verse vulnerables al momento de comparar sus efectos con esquemas de organización bajo modelos instruccionales específicos adecuados a las dimensiones y niveles de complejidad de conocimiento que se está instruyendo en cada caso. A su vez, la manera en que las programaciones contienen elementos instruccionales y estratégicos que posibiliten un desarrollo de habilidades de autorregulación para el aprendizaje aumenta de manera evidente tanto el rendimiento, el apego de los alumnos, y evita la deserción.

Otro de los elementos cruciales es el desarrollo de esquemas de trabajo que posibiliten no sólo la dosificación de actividades lineales ni de conocimiento plano. En este sentido, es necesario remarcar la idea de que la programación de actividades a realizarse en distintos niveles de complejidad y de esquemas mentales da diversidad al desarrollo cognitivo; por otra parte, la innovación al momento de generar tareas y recursos mediante modelos psicopedagógicos puede aumentar significativamente el logro académicos de los grupos. Resulta claro que de manera inicial los alumnos dependen mucho de la percepción que los demás tenemos acerca de ellos como estudiantes; sin embargo, es posible afirmar que dicha percepción puede irse 
canalizando a la búsqueda de la propia aprobación y autoevaluación, generando metas realistas y establecimiento compromisos que impliquen motivación y logro.

$\mathrm{Al}$ referirse el aspecto de la motivación, se pueden identificar las dos vías básicas: por una parte el hecho de remarcar la imagen individual ante el grupo que, aunque están en la distancia, pueden generarse espacios sociales en red que son determinantes al momento de la colaboración y el trabajo. A su vez, la otra vía corresponde a la generación de una identidad institucional a la cual sientan apego, esto por el hecho de que los alumnos estando en la distancia pueden sentir un alejamiento subjetivo de vida académica como tradicionalmente se entiende; sin embargo, a partir del uso de estrategias motivacionales que sean dirigidas al fomento de esa identidad universitaria, pueden generar un mayor apego que se traduce en permanencia y un mayor rendimiento.

Es necesario remarcar el papel que las plataformas virtuales adquieren. Para el caso del CUVED se puede decir que cuenta con recursos que permiten el registro, seguimiento y evaluación de una manera constante, además de tener una estabilidad en cuanto a sus sistemas y una variación de recursos didácticos que pueden ser ajustados a la necesidad instruccional y educativa del módulo y sus alumnos.

Evidentemente esta clase de investigaciones sugieren que la educación a distancia debiera enfocarse y promover la autonomía de los estudiantes en su propio proceso de aprendizaje. En este caso el CUVED fue programado para brindar recursos y espacios con base a funciones instruccionales de aprendizaje autorregulado y los estudiantes hicieron uso de estas funciones, aun cuando no fueron entrenados en habilidades autorregulatorias de aprendizaje, hallándose datos que ratifican tanto el desarrollo de estas habilidades como su relación en el impacto favorable que hay con respecto al rendimiento.

Aun con todo esto resulta importante señalar que la acción del docente (mejor denominado tutor para esta modalidad) no va en función al abandono progresivo o a restar importancia al desarrollo de los alumnos; por el contrario, su papel se centra desde el diseño y planeación, el seguimiento constante y la continua comunicación a fin de hacer efectiva la cualidad conducente del programa. En síntesis, resulta por demás señalar que intentar replicar la acción docente tradicional a un ambiente e-learning garantizará un fracaso rotundo, esto es, situarse a la incorporación de explicaciones iniciales largas, la encomienda de tareas simples y la ausencia de comunicación, sin mencionar la importancia de cuidar los indicadores de evaluación tanto sumativos como formativos. 
A modo de conclusión, es posible marcar que la conjunción de recursos es funcional y favorable. Para este caso el ambiente de aprendizaje de CUVED, con los recursos programados, las tareas planificadas y el diseño instruccional, jugaron un papel crucial en el desempeño de los alumnos. La acción educativa se dio desde la inclusión del alumno en un nuevo ambiente de aprendizaje, en el uso opcional de los recursos audiovisuales, la encomienda en la organización de sus propios tiempos, la evaluación basada en un Análisis Cognitivo de Tareas y en evaluaciones confiables, y la comunicación continua. Todo esto generando en conjunto una dinámica formativa que finalizó con mejores indicadores formativos en los estudiantes. Cada uno de estos elementos se vuelve importante para poder continuar con líneas de trabajo e investigación en el desarrollo de programas de educación a distancia con impacto.

Nuestro país está entrando en un periodo en donde la educación a distancia se convierte en algo más que una alternativa, pues está posibilitando el alcance de la educación, el aumento en la matrícula educativa, la reducción de costos, y la diversificación del conocimiento. Resulta así importante tomar en cuenta que su perfeccionamiento apenas comienza y que las líneas de trabajo pueden ser variadas e importantes para la solución de problemas educativos socialmente relevantes.

\section{REFERENCIAS BIBLIOGRÁFICAS}

Amador, L. (1998). Motivación en los universitarios a distancia. Mejorar el aprendizaje. RIED. Revista Iberoamericana de Educación a Distancia, 2 (1), (274).

Azevedo, R.; Winters, F. I.; Moos, D. C. (2004). Can students collaboratively use hypermedia to lean about science? The dynamics of self- and other-regulatory processes in an ecology classroom. Journal of Educational Computing Research, 31(3), (215-245).

Boekaerts, M.; Niemivirta, M. (2000). Selfregulated learning. Findinga balance between learning goals and egoprotective goals. En: Boekaerts, M.; Pintrichy, P. R.; Zeidner, M. Handbook of Self-Regulation. San Diego: Academic Press. (13-38).

Castañeda, S. (2004). Educación, aprendizaje y cognición. Guía abreviada del Modelo de Aprendizaje
Estratégico. México, DF: Manual Moderno.

Castañeda, S.; Martínez, R. (1999). Enseñanza y aprendizaje estratégicos. Modelo integral de evaluación e instrucción. Revista Latina de Pensamiento y Lenguaje, 4 (28), (251278).

Fernández, R.; Carballos, E.; Delavaut, M. (2008). Un modelo de autoaprendizaje con integración de las TIC'S y los métodos de gestión del conocimiento. RIED. Revista Iberoamericana de Educación a Distancia, 2 (11), (137149).

López, V. (2008). Desarrollo de la autorregulación en el aprendizaje con ambientes computacionales. Colombia: RIBIE.

Pintrichs, P. (2000). The role of goal orientation in self - regulated learning. En: Boekaerts, M.; Pintrichy, P. 
R.; Zeidner, M. Handbook of SelfRegulation. San Diego: Academic Press. (451-501).

Pintrich, P. R. (1995). Understanding SelfRegulated Learning. En: Pintrich, P.R. (Ed.). Understanding Self-Regulated Learning. New Directions for teaching and learning. San Francisco: JosseyBass.
Sarramona, J. (1999). La autoformación en una sociedad cognitiva. Revista Iberoamericana de Educación a Distancia, 1 (2), (41-59).

Zimmerman, B. J. (1986). A social cognitive view of self-regulated academic learning. Journal of Educational Psychology, 81 (3), (329-339).

\section{PERFIL ACADÉMICO Y PROFESIONAL DEL AUTOR}

Omar Moreno Almazán. Licenciado en Psicología desde 2001 por la UNAM. Terapeuta de adolescentes. Docente en la UNAM FES Iztacala en los Sistema Escolarizado y el Sistema de Educación a Distancia. Ha diseñado materiales didácticos digitales y de evaluación. Ponente en encuentros académicos nacionales e internacionales, y participante en eventos para instituciones de educación secundaria y preparatoria particulares y públicas, así como en instituciones gubernamentales. Miembro de la Sociedad Mexicana de Psicología, doctorando en Psicología por la UNAM, y jefe en el Sistema de Educación Abierta y Educación a Distancia de la carrera de Psicología, UNAM.

E-mail: almazanomar@gmail.com

DIRECCIÓN DEL AUTOR

FES Iztacala UNAM

Av. De los Barrios 1,

Los Reyes Iztacala;

Tlalnepantla, Estado de México.

Fecha de recepción del artículo: 01/09/11

Fecha de aceptación del artículo: 25/03/12

\section{Como citar este artículo:}

Moreno Almazán, O. (2012). Evaluación de un sistema instruccional autorregulatorio para un ambiente en línea: el caso de psicología en México. RIED. Revista Iberoamericana de Educación a Distancia, volumen 15, nº 2, pp. 75-94. 